ment for $A$, and since $B$ is simple, $A$ is also a direct sum of simple algebras.

\title{
REFERENCES
}

1. A. A. Albert, $A$ theory of power-associative commutative algebras, Trans. Amer. Math. Soc. 69 (1950), 503-527.

2. J. M. Osborn, Identities on nonassociative algebras, Canad. J. Math. 17 (1965), 78-92.

UNIVERSITY OF WISCONSIN

\section{SOME PROPERTIES OF LOCALIZATION AND NORMALIZATION}

\author{
JOSEPH LIPMAN
}

In a recent note [1], S. Abhyankar has given some lemmas concerning localization and normalization for noetherian rings without nilpotent elements. We give a characterization of those rings in which every prime ideal is maximal (Proposition 1) and deduce generalizations of Abhyankar's results (cf. Corollary 1 and Corollary 2).

Preliminaries. A ring will always be a nonnull commutative ring with identity.

For properties of rings of quotients see [3, $\$ \$ 9-11$ of Chapter IV]. Recall that if $R$ is a ring with total quotient ring $K$, and if $M$ is a multiplicative system in $R$, then we may identify the ring of quotients $R_{M}$ with a subring of $K_{M}$. When this is done, the total quotient ring of $K_{M}$ is also the total quotient ring of $R_{M}$.

Denote by $g_{M}$ the canonical map of $K$ into $K_{M}$; the restriction of this map to $R$ is then the canonical map of $R$ into $R_{M}$; the restricted map may also be denoted by $g_{M}$ without fear of confusion. If $M$ consists of all the powers of a single element $f$, then we write $R_{f}, K_{f}, g_{f}$, in place of $R_{M}, K_{M}, g_{M}$.

If $Q$ is a minimal prime ideal in $R$, and $M$ is the complement of $Q$ in $R$, then $Q R_{M}$, being the only prime ideal in $R_{M}$, consists entirely of zerodivisors (in fact, of nilpotents). Consequently, if $x \in Q$, then $g_{M}(x)$ is a zerodivisor, and it follows easily that $x$ is a zerodivisor. Thus any minimal prime ideal in a ring consists entirely of zerodivisors.

Proposition 1. For a ring $R$, the following statements are equivalent: (1) Every prime ideal in $R$ is maximal.

Received by the editors July 2, 1964. 
(2) For every multiplicative system $M$ in $R$, the canonical map $\mathrm{g}_{M}: R \rightarrow R_{M}$ is surjective.

(3) Any homomorphic image of $R$ is its own total quotient ring.

Proof. (1) $\rightarrow$ (3). If $\bar{R}$ is a homomorphic image of $R$, then every prime ideal in $\bar{R}$ is maximal; therefore every prime ideal in $\bar{R}$ is minimal and so consists entirely of zerodivisors. It follows that every non-unit in $\bar{R}$ is a zerodivisor; (3) is thereby proved.

$(3) \rightarrow(2)$ is clear, since $R_{M}$ is contained in the total quotient ring of $g_{M}(R)$.

$(2) \rightarrow(1)$, for if $P<Q$ are proper prime ideals, ( $<$ means "strictly contained in") and if $f$ is any element in $Q-P$, then $P$ contains the kernel of $g_{f}: R \rightarrow R_{f} ;$ a fortiori, $Q$ contains the kernel, whence $g_{f}(Q)$ is a proper ideal in $g_{f}(R)$. But $g_{f}(f) \in g_{f}(Q)$ is a unit in $R_{f}$; hence $g_{f}(R)<R_{f}$.

COROLlaRY 1. For a ring $R$ with total quotient ring $K$, the following statements are equivalent:

(1) Every prime ideal in $R$ consisting entirely of zerodivisors is a minimal prime ideal.

(2) For every multiplicative system $M$ in $R, g_{M}(K)$ is the total quotient ring of $R_{M}$.

Proof. In view of the relationship between the prime ideals in $R$ and those in the total quotient ring of $R,(1)$ implies that every prime ideal in $K$ is maximal. By the proposition, this means that $K_{M}$ $=g_{M}(K)$ is its own total quotient ring, and so (2) holds.

Conversely, if $P<Q$ are prime ideals in $R$ consisting entirely of zerodivisors, and if $f \in Q-P$ then $P K<Q K$ are proper prime ideals in $K, f \in Q K-P K$, and, as in the proof of Proposition $1, g_{f}(K)<K_{f}$ $\subseteq$ total quotient ring of $R_{f}$; hence (2) does not hold.

EXAmples. The conditions of the corollary are satisfied by any noetherian ring in which the ideal (0) has no embedded primes; in particular, by any noetherian ring without nilpotent elements $[3$, \$5-6 of Chapter IV].

Another example is the following: Let $k$ be a field, $R$ and $R^{\prime}$ two integral domains containing $k$. Then $R \otimes_{k} R^{\prime}$ satisfies the conditions [3, p. 191].

The conductor. Let $B$ be a ring, let $A$ be a subring of $B$, and let $\bar{A}$ be the integral closure of $A$ in $B$. If $M$ is a multiplicative system in $A$, then, after suitable identifications are made, $A_{M} \subseteq \bar{A}_{M} \subseteq B_{M}$, and by $\left[2\right.$, p. 22] $\bar{A}_{M}$ is the integral closure of $A_{M}$ in $B_{M}$.

The conductor $\subseteq(A, B)$ of $A$ in $B$ is defined to be the annihilator of 
the $A$-module $\bar{A} / A$. $\subseteq(A, B)$ is an ideal in $A$. If $c \in \Subset(A, B)$ then $c \bar{A} \subseteq A$, whence $c \bar{A}_{M} \subseteq A_{M}$, so that $c \in \mathbb{E}\left(A_{M}, B_{M}\right)$; thus $\mathbb{E}(A, B) \cdot A_{M}$ $\subseteq \mathfrak{E}\left(A_{M}, B_{M}\right)$. In particular if $\mathbb{E}(A, B) \cdot A_{M}=A_{M}$ then $\mathbb{E}\left(A_{M}, B_{M}\right)$ $=A_{M}$; hence if $\mathbb{S}(A, B)$ meets $M$ then $A_{M}$ is integrally closed in $B_{M}$.

COROLLARY 2. If $R$ is a ring in which every prime ideal consisting of zerodivisors is minimal, if $K$ is the total quotient ring of $R$, and if $M$ is a multiplicative system in $R$ such that $M$ meets the conductor $\mathbb{E}(R, K)$, then $R_{M}$ is integrally closed in its total quotient ring.

Proof. By Proposition 1 and Corollary $1, K_{M}=g_{M}(K)=$ the total quotient ring of $R_{M}$. We may therefore apply the preceding remarks with $A=R, B=K$.

\section{REFERENCES}

1. S. Abhyankar, $A$ remark on the nonnormal locus of an analytic space, Proc. Amer. Math. Soc. 15 (1964), 505-508. (Cf. also Correction, ibid., p. 1000).

2. N. Bourbaki, Algèbre commutative, Ch. 5-6, Actualités Sci. Ind. No. 1308, Hermann, Paris, 1964.

3. O. Zariski and P. Samuel, Commutative algebra, Vol. I, Van Nostrand, Princeton, N. J., 1958.

HARVARD UNIVERSTTY 